Case Report

\title{
ANCA Associated Vasculitis and Renal Failure Related to Propylthiouracil and Hyperthyroidism Induced Cholestasis in the Same Case
}

\author{
Mehmet Tuncay, Emine Kivrakoglu, Itir Yegenaga, and Erkan Dervisoglu \\ Department of Internal Medicine, Medical School Kocaeli University, 41380 Kocaeli, Turkey \\ Correspondence should be addressed to Itir Yegenaga; itiryegenaga@hotmail.com
}

Received 5 May 2014; Accepted 15 October 2014; Published 20 November 2014

Academic Editor: Sofia Lionaki

Copyright (C) 2014 Mehmet Tuncay et al. This is an open access article distributed under the Creative Commons Attribution License, which permits unrestricted use, distribution, and reproduction in any medium, provided the original work is properly cited.

\begin{abstract}
Introduction. Liver involvement due to hyperthyroidism and also ANCA positive vasculitis related renal failure cases were reported separately several times before. However, to our knowledge, these two complications together in the same case had never been observed before. Case Presentation. The case of an ANCA positive 71-year-old Caucasian male with renal failure and lung involvement, subclinical hyperthyroidism, and intrahepatic cholestatic jaundice was presented in this paper. After exclusion of all of the other possibilities, cholestatic hepatitis was explained by subclinical hyperthyroidism; renal failure and lung involvement were interpreted as ANCA related vasculitis which might be a side effect of propylthiouracil use. Conclusion. The coexistence of these rare conditions in the same patient deserves emphasis and it is worth reporting. This case demonstrates that following the clinical course of the patient is essential after prescribing any medications to see whether any complication occurs or not. If the complications of this case were noticed earlier, it would be possible to treat and to prevent the permanent damages.
\end{abstract}

\section{Introduction}

It was reported previously that at least one hepatic abnormality was observed in $60.5 \%$ of the patients with hyperthyroidism because of hypoxia in hepatocytes [1]. Increased metabolic rate in hyperthyroidism causing extra demand and insufficiency of oxygen demand in hepatocytes was claimed from these abnormalities $[2,3]$.

Propylthiouracil (PTU) is one of the most common drugs to treat hyperthyroidism which is reported to be responsible for ANCA (anti-neutrophil cytoplasmic autoantibody) positivity in $4 \%$ to $46 \%$ of cases, and even leading to vasculitis especially in China and Japan [4]. Even though PTU induced ANCA positive vasculitis cases were reported more often in Grave's disease, it also may be related to multinodular toxic goiter [5]. PTU-induced ANCA associated systemic vasculitis (AASV) was reported to be involved with the kidneys, skin, and pulmonary system; furthermore, arthralgia and fever were also observed as the most common symptoms [6]. Kidney involvement was reported to be present from mild to severe degree [7].
Based on these information, this case was found to be interesting and rare to be presented here. The case was interpreted as intrahepatic cholestasis caused by nodular toxic goitre and the drug which was used to treat hyperthyroidism leading to ANCA related vasculitis and renal failure.

\section{Case Presentation}

A 71-year-old Caucasian male was admitted to the outpatient nephrology clinic with the complaints of general malaise, progressive asthenia, weight loss, loss of appetite, jaundice, and decrease in urine volume. On admission, blood pressure was $120 / 70 \mathrm{~mm} / \mathrm{Hg}$; the pulse rate was regular $95 / \mathrm{min}$. $\mathrm{He}$ was pale, icteric and dehydrated. On physical examination, no abnormalities were found except hepatosplenomegaly and also multiple nodules in his thyroid glands. He had a history of subclinical hyperthyroidism and hypertension for about 30 years and treated with PTU irregularly, carvedilol $(12.5 \mathrm{mg} /$ day $)$, and trimetazidine $(60 \mathrm{mg} /$ day $)$. The patient was hospitalized for further investigation. 
TABLE 1: Laboratory findings of the case.

\begin{tabular}{|c|c|c|c|c|}
\hline & The day of admission & First dialysis & RAI treatment & Normal values \\
\hline Hemoglobin (g/dL) & 8.97 & 9.49 & 7.84 & $12.2-18.1$ \\
\hline Hematocrit (\%) & 26.3 & 27.5 & 23.4 & $37.7-53.7$ \\
\hline Neutrophil $\left(\times 10^{3} / \mathrm{mL}\right)$ & 1.470 & 1.930 & 1.350 & $4.60-10.2$ \\
\hline Thrombocyte $\left(\times 10^{3} / \mathrm{mL}\right)$ & 135 & 118 & 8.4 & $142-424$ \\
\hline Ferritin & 558 & & & $20-400$ \\
\hline Erythrocyte sedimentation rate $(\mathrm{mm} / \mathrm{h})$ & 82 & 45 & 42 & $0-20$ \\
\hline $\mathrm{C}$ reactive protein $(\mathrm{mg} / \mathrm{dL})$ & 5.34 & 11.4 & 3 & $0-0.5$ \\
\hline Glucose (mg/dL) & 114 & 116 & 134 & $70-105$ \\
\hline Urea (mg/dL) & 137 & 254 & 160 & $15-55$ \\
\hline Creatinine $(\mathrm{mg} / \mathrm{dL})$ & 2.8 & 3.87 & 3.88 & $0.6-1.53$ \\
\hline Uric acid (mg/dL) & 9.7 & 6.9 & 5.1 & $2.5-7.7$ \\
\hline Arterial pH & 7.3 & 7.1 & 7.4 & $7.35-7.45$ \\
\hline $\mathrm{HCO}_{3}(\mathrm{mmol} / \mathrm{L})$ & 15.6 & & & $22-26$ \\
\hline Direct bilirubin (mg/dL) & 2.2 & 8.1 & 9 & $0.00-0.50$ \\
\hline Aspartate aminotransferase (U/L) & 67 & 34 & 58 & $5-34$ \\
\hline Alanine aminotransferase (U/L) & 58 & 83 & 59 & $0-55$ \\
\hline Gamma glutamyl transferase (U/L) & 3 & & & $9-64$ \\
\hline Lactate dehydrogenase (U/L) & 127 & 132 & 127 & $125-245$ \\
\hline Alkaline Phosphatase (U/L) & 1290 & 1049 & 932 & $40-150$ \\
\hline T. protein $(\mathrm{g} / \mathrm{dL})$ & 6.8 & 6 & 5.5 & $6.4-8.3$ \\
\hline Calcium (mg/dL) & 8.5 & 8.5 & 7.9 & $8.4-10.2$ \\
\hline I. phosphorus (mg/dL) & 5.3 & 6.5 & 3.1 & $2.7-4.5$ \\
\hline Potassium $(\mathrm{mEq} / \mathrm{L})$ & 5.2 & 5.1 & 3.3 & $3.5-5.1$ \\
\hline Chlorine $(\mathrm{mEq} / \mathrm{L})$ & 110 & 115 & 104 & $98-107$ \\
\hline Parathyroid hormone (pg/mL) & 56.2 & & & $12-88$ \\
\hline Free $\mathrm{T}_{3}(\mathrm{pg} / \mathrm{mL})$ & 3.2 & 8.63 & 2.12 & $2.3-4.2$ \\
\hline Free $\mathrm{T}_{4}(\mathrm{ng} / \mathrm{dL})$ & 1.25 & 2.29 & 0.70 & $0.74-1.52$ \\
\hline Thyroid stimulating hormone (mIU/L) & 0.014 & 0.011 & 0.547 & $0.55-4.78$ \\
\hline
\end{tabular}

As it is shown in Tables 1 and 2, he showed normokrom normositer anemia pancytopenia, cholestasis, and subclinical hyperthyroidism in his laboratory examination.

Ultrasonographic examination and magnetic resonance imaging (MRI) of abdomen were performed to exclude extrahepatic cholestasis and showed no clues for malignancies but heterogeneity of liver parenchyma; intra- and extrahepatic bile duct sizes were normal; enlargement of liver $(170 \mathrm{~mm})$ and spleen $(188 \mathrm{~mm})$ were observed; in addition, portal and splenic vein diameters were increased. Heart failure and the other rare causes of intrahepatic cholestasis were also excluded. Pathological examination of liver biopsy showed that there was sparse piece-meal necrosis and inflammatory cell infiltration in the portal areas. Focal ductuli proliferation, pericentral intracytoplasmic bile pigments, bile plugs, and diffuse focal necrosis were also noted (Figure 1). Histopathological findings were interpreted as intrahepatic cholestasis might be related to thyrotoxicosis.

Serological markers, autoantibodies, laboratory findings, and liver biopsy findings helped us to exclude primary biliary cirrhosis, autoimmune hepatitis, Wilson's disease, hemochromatosis, and viral hepatitis.

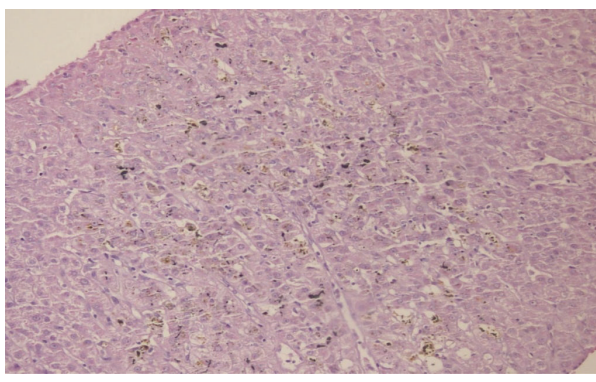

Figure 1: Biopsy material of liver.

On his 14th day of hospitalization, tachycardia, elevated blood pressure, tachypnea, agitation, and dysphonia were observed. His renal function was deteriorated and hemodialysis treatment was initiated.

Since PTU and methimazole treatments have been reported to be related with AASV, we decided to perform radioactive iodine $\left({ }^{131} \mathrm{I}-10 \mathrm{mCi}\right)$ treatment on 27 th day of admittance for hyperthyroidism. After the RAI treatment, 
TABLE 2: Immunologic parameters of the case.

\begin{tabular}{|c|c|c|}
\hline & The day of admission & Normal values \\
\hline CA 125 (OM-MA) (U/mL) & 9.58 & $0.0-30.2$ \\
\hline CA 19-9 (GI-MA) (U/mL) & 13.1 & $0.0-35$ \\
\hline Carcinoembryonic antigen (ng/mL) & 0.18 & $0.0-0.25$ \\
\hline Antithyroidperoxidase (IU/mL) & 35.8 & $0.0-60$ \\
\hline Antithyroglobulin (IU/mL) & 21.5 & $0.0-60$ \\
\hline Alpha fetoprotein (IU/mL) & 1.03 & $0.5-5.0$ \\
\hline Antinuclear antibody & +3 Nuclear membrane & \\
\hline Antinuclear cytoplasmic antibody (MPO) & +2 & \\
\hline Antinuclear cytoplasmic antibody elastase & +2 & \\
\hline Cytoplasmic antinuclear cytoplasmic antibody & $( \pm)$ & \\
\hline Antimitochondrial antibody & $(-)$ & \\
\hline Anti-liver kidney microsome antibody & $(-)$ & \\
\hline HBs Ag (S/CO) & $<-1.0(-)$ & \\
\hline Anti-HBs (IU/L) & $<-10.0(-)$ & \\
\hline Anti-HBcIgG (S/CO) & $<-1.0(-)$ & \\
\hline $\mathrm{HBe} \mathrm{Ag}(\mathrm{S} / \mathrm{CO})$ & $<-1.0(-)$ & \\
\hline Anti-HBe (S/CO) & $>-1.0(-)$ & \\
\hline Anti-HAV IgG (S/CO) & $>-1.0(+)$ & \\
\hline Anti-HAV IgM (COI) & $<-1.0(-)$ & \\
\hline Anti-HCV (S/CO) & $<-1.0(-)$ & \\
\hline
\end{tabular}

HBs Ag: hepatitis B surface antigen, Anti-HBs: anti-hepatitis B surface antibody, Anti-HBe: anti-hepatitis e antigen, Anti-HAV IgG: anti-hepatitis A virus immunoglobulin G, Anti-HAV IgM: anti-hepatitis A virus immunoglobulin M, and Anti-HCV: anti-hepatitis C virus.

patient became euthyroid but cholestasis was not ameliorated. Ursodeoxycholic acid (1500 $\mathrm{mg} /$ day) and rifampicin $(600 \mathrm{mg} /$ day $)$ treatments also did not bring any further benefit in point of cholestasis.

Patient was pancytopenic on his admittance and during the follow-up; haematological examination and bone marrow biopsy showed no significant pathology. Serum and urine immune fixation electrophoresis were performed to exclude multiple myeloma, a possible cause of anemia/pancytopenia in the elderly. Moreover, rectal biopsy was negative for the amyloidosis, which might be possible explanation for the hepatosplenomegaly, kidney failure, and pancytopenia as well. And it was concluded that pancytopenia might be related to hypersplenism.

Because the patient had suffered from dysphonia and dyspnea, thorax computerized tomography (CT) was performed and several nodules were discovered in right lung parenchyma (Figure 2); the right apicoposterior one was cavitary; nevertheless, pneumonia, tuberculosis, and malignancies were excluded. These findings supported lung involvement of ANCA positive vasculitis.

After exclusion of all of these possibilities, we concluded that the patient was suffering from hyperthyroidism induced intrahepatic cholestasis. Furthermore, since hyperthyroidism was not taken under control for many years, the changes in the liver became permanent and leading to developed cirrhosis eventually. In addition, we thought that using PTU treatment irregularly to control hyperthyroidism caused ANCA positive vasculitis with renal and lung involvement.

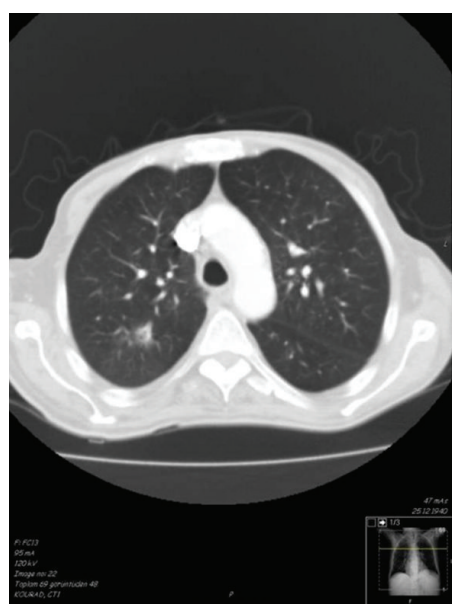

FIGURE 2: Computerized tomography of lung.

\section{Discussion}

Although during the clinical course of hyperthyroidism mild changes in liver functional tests are often observed, it is reported that in untreated hyperthyroidism pathological changes in liver might progress into focal necrosis and centrilobular intrahepatic cholestasis and eventually cirrhosis can also develop [2]. Nevertheless, subclinical hyperthyroidism induced intrahepatic cholestasis cases like the case presented here are rarely observed [3]. 
ANCA is usually formed against the serine proteases in monocytes and neutrophils, identified by indirect immunofluorescence. Even though it is not well known, mechanism of PTU-induced AASV is explained as transforming of MPO (myeloperoxidase) into sulfonate by PTU which is highly antigenic and induces autoimmunity. Activated T-lymphocytes stimulate ANCA production by B-lymphocytes and causes vascular injury [8]. It was also reported that higher titration of MPO-ANCA might be the most important risk factor for the development of systemic vasculitis [9]. It was also reported that anti-MPO antibody titration decreases with the immune suppressive therapy or cessation of PTU treatment [10].

In this case, ultrasonographic examination showed small kidneys compatible with chronic kidney failure on admittance; therefore, renal biopsy could not be performed but it was postulated that kidney involvement might be related to vasculitis. However, histopathological examination of kidney in PTU induced that AASV was reported as necrotizing and crescentic pauci-immune glomerulonephritis and total sclerosis [7].

The most common lung involvement in PTU-induced AASV has been reported as diffuse alveolar hemorrhage [11]. But rarely multiple nodules in chest CT were determined similarly in this case [12].

In our case, there were no skin lesions observed but purpuric, erythematous, and necrotic skin lesions were commonly reported in PTU-induced AASV with renal and lung involvement [6] and histopathological examination of these skin biopsy materials showed leukocytoclastic and fibrinoid necrosis [6].

\section{Conclusion}

This case, presented here, carried multiple complications altogether which is interesting and very rare and also we wanted to emphasize that, to follow-up, the results of treatments are very important to see the side effects and complications. If we notice them earlier, it is possible to correct and save the patient from a lot of burden.

\section{Abbreviations}

PTU: Propylthiouracil

ANCA: Anti-neutrophil cytoplasmic autoantibody

AASV: ANCA associated systemic vasculitis

MRI: Magnetic resonance imaging

CT: Computerized Tomography

MPO: Myeloperoxidase.

\section{Consent}

Written informed consent was obtained from the patient for publication of this case report and any accompanying images. A copy of the written consent is available for review.

\section{Conflict of Interests}

The authors declare that they have no competing interests.

\section{Authors' Contribution}

Mehmet Tuncay supervised the follow-up of the case and commented on the symptoms, signs, and laboratory tests. Emine Kivrikoglu assisted in following up on the tests and organizing the treatment of the case. Itir Yegenaga collected the data and wrote the paper. Erkan Dervisoglu helped to organize data and write it down.

\section{Acknowledgments}

The authors appreciate the kind contribution of Radiology Department and Pathology Department of Kocaeli University Medical School and Professor Dr. Yesim Gurbuz.

\section{References}

[1] A. Gürlek, V. Çobankara, and M. Bayraktar, "Liver tests in hyperthyroidism: effect of antithyroid therapy," Journal of Clinical Gastroenterology, vol. 24, no. 3, pp. 180-183, 1997.

[2] R. Malik and H. Hodgson, "The relationship between the thyroid gland and the liver," QJM, vol. 95, no. 9, pp. 559-569, 2002.

[3] J.-F. Viallard, A. Tabarin, D. Neau, and M. Longy-Boursier, "Hyperthyroidism with severe intrahepatic cholestasis," Digestive Diseases and Sciences, vol. 44, no. 10, pp. 2001-2002, 1999.

[4] M. C. Slot, T. P. Links, C. A. Stegeman, and J. W. C. Tervaert, "Occurrence of antineutrophil cytoplasmic antibodies and associated vasculitis in patients with hyperthyroidism treated with antithyroid drugs: a long-term followup study," Arthritis Care and Research, vol. 53, no. 1, pp. 108-113, 2005.

[5] N. Ş. Y. Bilge, T. Kaşifoğlu, and C. Korkmaz, "PTU-induced ANCA-positive vasculitis: an innocent or a life-threatening adverse effect?" Rheumatology International, vol. 33, no. 1, pp. 117-120, 2013.

[6] R. A. Quax, A. J. Swaak, and M. G. Baggen, "Churg-Strauss syndrome following PTU treatment," International Journal of Rheumatology, vol. 2009, Article ID 504105, 4 pages, 2009.

[7] F. Yu, M. Chen, Y. Gao et al., "Clinical and pathological features of renal involvement in propylthiouracil-associated ANCApositive vasculitis," The American Journal of Kidney Diseases, vol. 49, no. 5, pp. 607-614, 2007.

[8] S. von Schmiedeberg, C. Goebel, E. Gleichmann, J. Uetrecht, and R. L. Rubin, "Neutrophils and drug metabolism," Science, vol. 268, no. 5210, pp. 585-586, 1995.

[9] H. Ye, Y. Gao, X.-H. Guo, and M.-H. Zhao, “Titre and affinity of propylthiouracil-induced anti-myeloperoxidase antibodies are closely associated with the development of clinical vasculitis," Clinical \& Experimental Immunology, vol. 142, no. 1, pp. 116-119, 2005.

[10] D. R. W. Jayne, G. Gaskin, C. D. Pusey, and C. M. Lockwood, "ANCA and predicting relapse in systemic vasculitis," Quarterly Journal of Medicine, vol. 88, no. 2, pp. 127-133, 1995.

[11] K. Yamauchi, M. Sata, J. I. Machiya et al., "Antineutrophil cytoplasmic antibody positive alveolar haemorrhage during 
propylthiouracil therapy for hyperthyroidism," Respirology, vol. 8, no. 4, pp. 532-535, 2003.

[12] N. Shimizudani, S. Senba, T. Odaka et al., "A case of MPOANCA positive vasculitis associated with multiple pulmonary nodules following propylthiouracil treatment," Nihon Kokyūki Gakkai Zasshi, vol. 47, no. 2, pp. 98-103, 2009. 


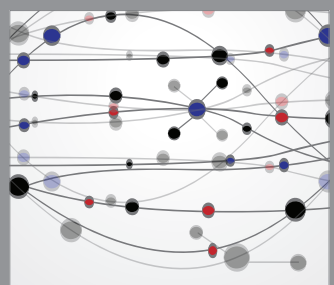

The Scientific World Journal
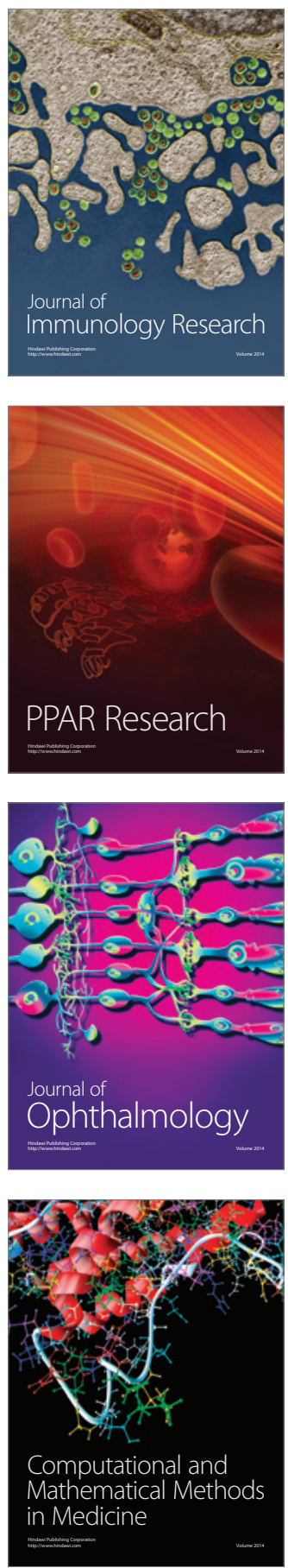

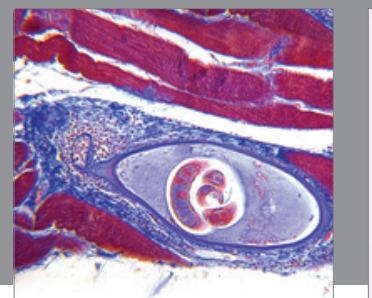

Gastroenterology

Research and Practice
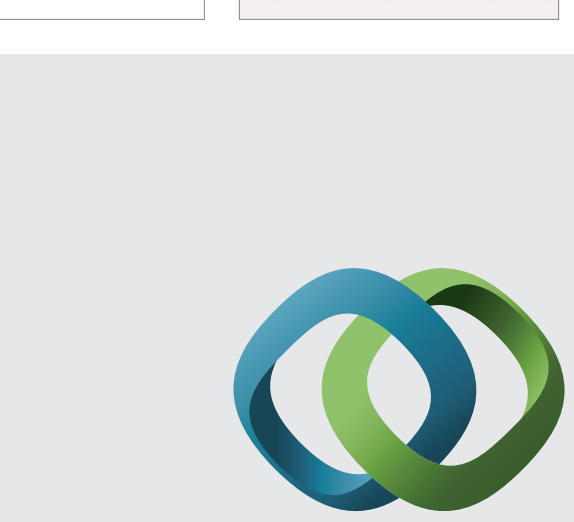

\section{Hindawi}

Submit your manuscripts at

http://www.hindawi.com
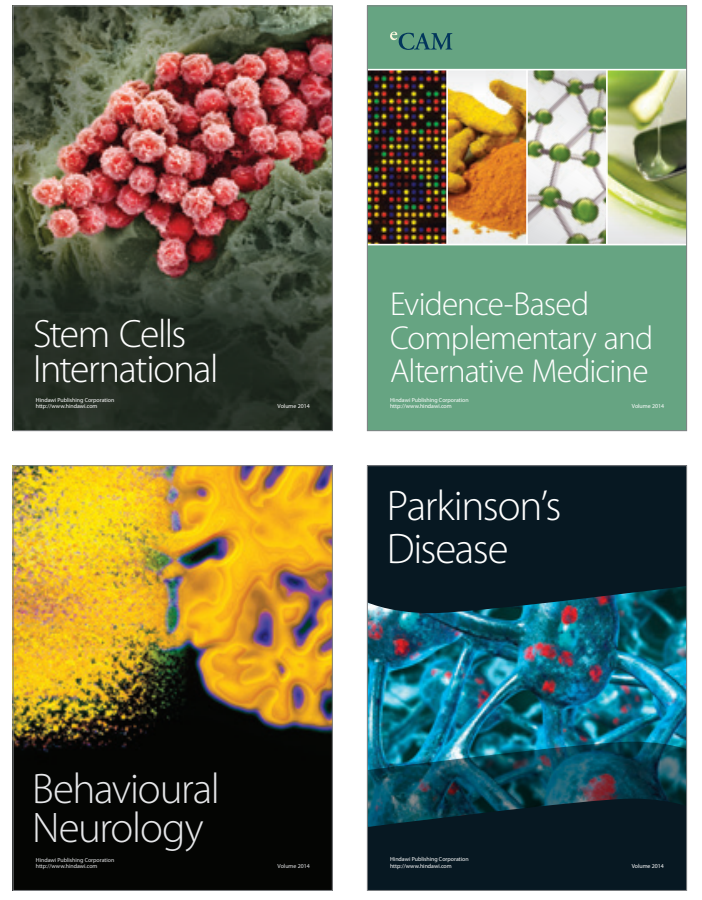
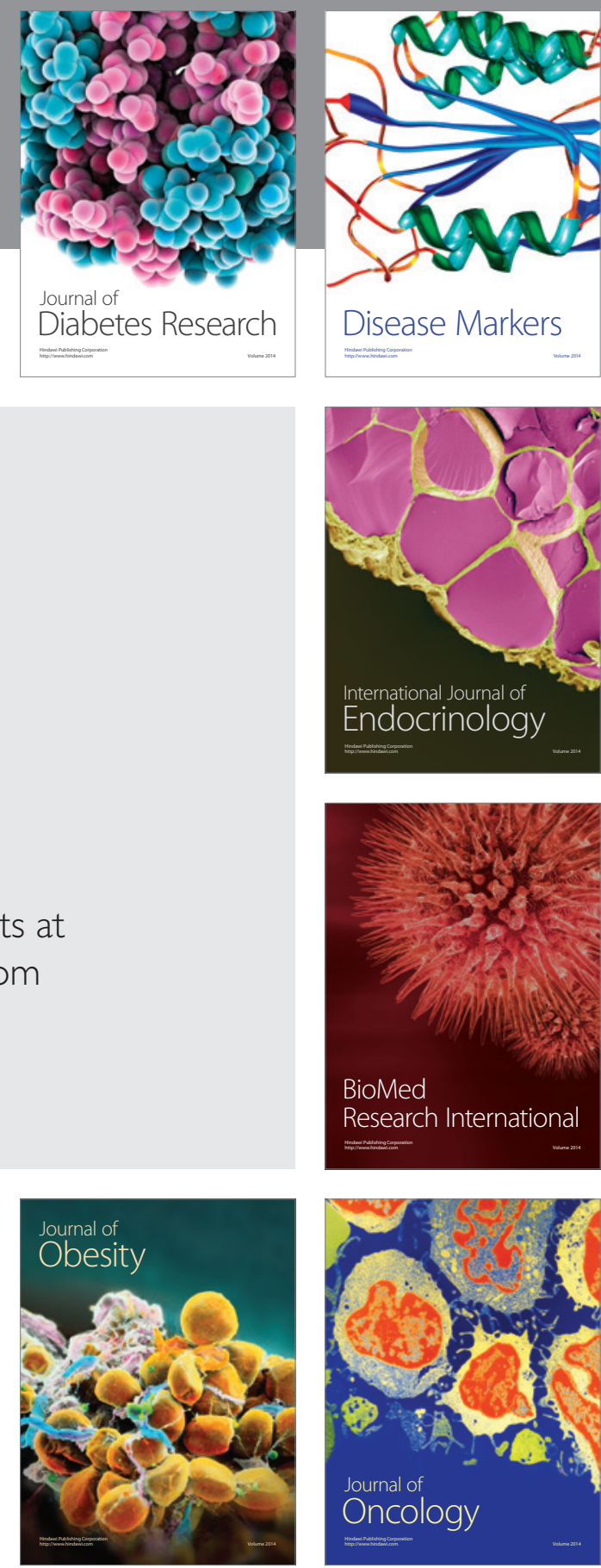

Disease Markers
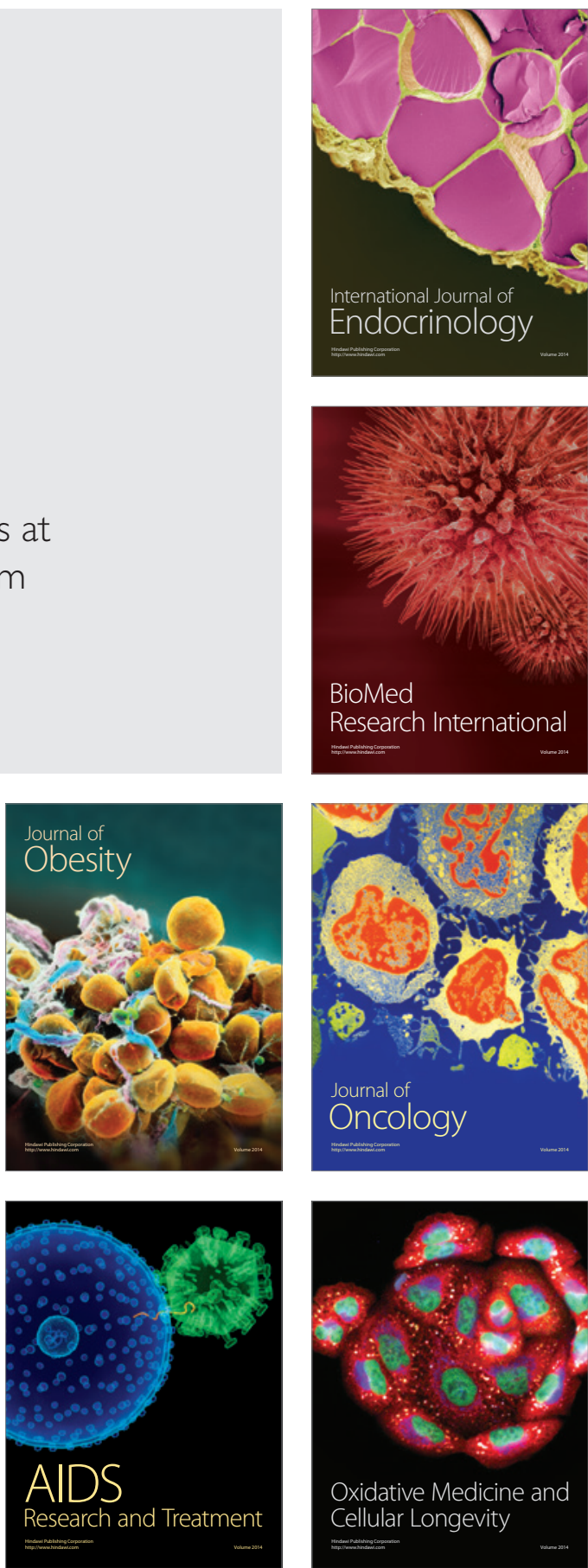